\title{
Rare case of Bicornuate Uterus with Fetal Death in a Multipara woman
}

\author{
Alen Kinyina \\ Open University of Tanzania (OUT), Dar es salaam-Tanzania
}

\begin{abstract}
Congenital uterine anomalies result from defect in unification of the müllerian duct during intrauterine fetal life. Pregnancy in such abnormalities associated with poor outcome including rudimentary horn rupture, miscarriage, hemorrhage and death. The diagnosis is also difficult in lower resource settings and case management requires good skills in surgery. We present a case with failed labour induction and misdiagnosis of pregnancy in rudimentary horn of bicornuate uterus. This case has tremendously delay in diagnosis and management, however the final decision of conducting surgery was implicit successful.
\end{abstract}

Keywords: Bicornuate, Rudimentary horn, Uterus.

\section{INTRODUCTION}

Most Bicornuate uterus and other types of congenital uterine malformations results from abnormal fusion or resorption of the paramesonephric duct (müllerian duct) during embryogenic development. ${ }^{[1]}$ There is no causal relationship between Müllerian anomalies and infertility, ${ }^{[2]}$ although Pregnancy in that anomalies is rare and occurs in approximately $1: 76,000$ pregnancies $^{[3]}$

Women with uterine abnormalities have high risk of miscarriage, poor fetal growth, malpresentations, ectopic pregnancies, hemorrhage, fetal and maternal mortality. ${ }^{[1,3,]}$ Pregnancy in rudimentary horn of uterus has high risk of rupture occurring in approximately $50 \%$ of pregnancies, mostly before the third trimester. ${ }^{[3,4]}$

Sensitivity of ultrasound in diagnosing rudimentary horn is only $26 \%$ and effectiveness of diagnosis before rupture is $14 \%$ depending on the skills of operator. ${ }^{[2,3,5]}$ Hence misdiagnosis is common as how it happened in our case. We therefore report this case due to its rarity.

\section{CASE REPORT}

A 21 years old woman Gravida 3 Para 2 referred from Primary health care (PHC) center to Public District Hospital. The patient has history of two spontaneous vaginal deliveries and all 2 children are alive, she has no abortion or surgical history. Menstrual cycles were normal with regular flow. At time of admission she was complaining of abdominal pain and absence of fetal movements of unknown duration, in a pregnancy of 23 weeks' gestation.

The abdominal pain started 4 hours prior to referral. It was diffuse, of moderate intensity and radiating to back. Per abdominal examination there was a palpable mass below the umbilicus and with no fetal tone on auscultation. Ultrasound was done and confirmed a presence of a dead fetus in the uterus with decreased amniotic fluid.

The patient received two doses of Misoprostol 200 microgram intravaginally with 4 hours apart between doses administration for labour induction. After labour induction failure on the first day, the dose was repeated on the second day with no any progress on the state of cervix or signs of labour. It was decided to stop labour induction with Misoprostol and keep the patient under ant-pain and observation while waiting for spontaneous labour.

Ten days later the labour induction was repeated under normal principles and protocols of labour induction in intrauterine fetal death without success. Ultrasound was done and re-confirmed a presence of a dead fetus in the uterus with decreased amniotic fluid.

Nine days later, under general anesthesia dilatation and curettage $(\mathrm{D} \& \mathrm{C})$ was done; some endometrial tissue came out of uterus with no any product of conception, the procedure of $\mathrm{D} \& \mathrm{C}$ was ceased to avoid trauma. The patient was complaining of severe abdominal pain with moderate per vaginal bleeding. Laparotomy was done immediately; and a 23 weeks' dead fetus was found in the left ruptured rudimentary horn of bicornuate uterus, the right normal horn was perforated during D\&C. Dead fetus was extruded followed by ablation of ruptured rudimentary horn and fallopian tube. Bleeding was controlled, recovery was uneventful and a patient was discharged after seven days.

\section{DISCUSSION}

Müllerian anomalies are rare cases and always remain asymptomatic till fortune imaging procedures or during surgery as how it happened in our case. ${ }^{[2,6]}$ To our knowledge and a review of the literatures no case has been reported in Tanzania. Usually ultrasonography highly depends on operator's skills, due to that reason many cases of bicornuate uterus and rudimentary uterine horn pregnancies are missed like in our case. ${ }^{[5,7]}$

Ultrasonography of abdomen was done, although doctor gave the report as normal, probably due to lack of experience. An expert in ultrasonography can detect some cases of müllerian abnormalities, although it requires magnetic resonance imaging (MRI) and CT Scan to confirm. ${ }^{[5,8]}$ 
In our case the fetus was not alive however the management was tremendously missed. Induction of labor with misoprostol in rudimentary horn pregnancies is highly discouraged due to increased high risk of rupture. ${ }^{[2]}$ Under normal circumstance cautious and early management of Intrauterine fetal death (IUFD) is recommended due to high risks of intrauterine infections and time-related risk of consumptive coagulopathy. [9] Rudimentary horn pregnancies in both viable and non-viable fetus are best managed by surgery. ${ }^{[5,10]}$

\section{CONCLUSION}

It is important to conduct careful evaluation of reproductive tract with additional imaging clues to have correct diagnosis; and rule out several causes of failed management because poor or wrong management can culminate in poor outcome.

\section{ACKNOWLEDGMENT}

The author would like to acknowledge the contribution of Mwarabu Chowo, Daud Philipo, Justine Samson, Yohana Masubo, Morad Faustine, Mpanuka Dieudonne, MSF Midwives Nduta, Maternity and ObGy team of $\mathrm{KDH}$ and Bernard Gwaho.

\section{REFERENCES}

[1]. Madhavi D (2012). Bicornuate Uterus-A Case Report. Journal of Anat Physiol 2:109

[2]. Florent Ymele et al, Spontaneous second-trimester ruptured pregnancy of rudimentary horn: a case report in Yaounde, Cameroon. The Pan African Medical Journal. 2014; 18:86

[3]. Emma Torbe, Mei-See Hon (2012). Subsequent pregnancy after a ruptured rudimentary uterine horn pregnancy.European journal of Obstetrics and Gynacology and Reproductive Biology : Volume 166 issue 1.

[4]. Kathpalia S. Rudimentary horn - different clinical presentations. Obstet Gynecol Int J. 2018;9(6):440 442.

[5]. Gary Sutkin, Allahyar Jazayeri. Diagnosis of a Rudimentary Uterine Horn in Pregnancy. J Ultrasound Med 22:985-988, 2003

[6]. Lacin S, Kartal O. Rupture of rudimentary horn pregnancy at the 15 th week of gestation: a case report. Eur J Obstet Gynecol Reprod Biol. 2002;102(2):209221.

[7]. Goel P, Saha PK, Mehra R, Huria A. Unruptured postdated pregnancy with a live fetus in a noncommunicating rudimentary horn. Indian $\mathrm{J}$ Med Sci. 2007; 61 (1): 23-7.

[8]. Tsafrir A, Rojansky N, Sela HY, Gomori JM, Nadjari M. Rudimentary horn pregnancy: first-trimester prerupture sonographic diagnosis and confirmation by magnetic resonance imaging. J Ultrasound Med. 2005; 24(2):219-23
[9]. Patel S et al. Int J Reprod Contracept Obstet Gynecol. 2014 Dec;3(4):931-935Study of causes and complications of intra uterine fetal death (IUFD)

[10]. Rackow BW, Arici A. Reproductive performance of women with müllerian anomalies. Curr Opin Obstet Gynecol. 2007; 19(3):229-37. 